\title{
Intelligent Dumbbell Based on Multiple Sensors
}

\author{
Yang $\mathrm{Liu}^{1 *}$ and Chun-Liang $\mathrm{Hsu}^{2}$ \\ ${ }^{1}$ Department of Electronic Engineering, Dongguan Polytechnic, No. 3, University Rd., \\ Song-Shang Lake District, Dongguan, Guangdong 523808, China \\ ${ }^{2}$ Department of Electronic Engineering, St. John's University, \\ No. 499, Sec. III, Tan-Kin Rd., Tan-Shuei District, New Taipei City 25135, Taiwan
}

(Received November 30, 2019; accepted April 28, 2020)

Keywords: accelerometer, thin-film pressure sensor, microprocessor, wireless communication, motion monitoring

In recent years, with the economic development, people's living standards have continuously improved, and obesity, suboptimal health, and other issues have continued to appear and have become the focus of people's attention. To maintain a healthy body, more people are participating in fitness exercises. However, traditional fitness equipment can be boring and inflexible in its use and does not enable the recording of fitness data, making it difficult for people to monitor fitness data and manage their health information. The design of miniaturized intelligent equipment with comprehensive management will be of great significance in meeting the fitness needs of users and have a wide range of applications. As small fitness equipment, dumbbells are suitable for people of different ages, genders, and physical fitness levels. To increase the fun of dumbbell fitness and realize guidance in dumbbell fitness exercise, we have designed an intelligent dumbbell based on multiple-sensor information fused with wireless communication technology. The intelligent dumbbell can automatically recognize its weight through a pressure sensor and judge fitness exercise through an acceleration sensor. The information is then transmitted by a wireless transmission module to a mobile phone application (app) to realize the intelligent monitoring of dumbbell fitness. At the same time, the user can also set the fitness mode through the mobile phone app to guide the user in exercise and more systematically improve the user's fitness.

\section{Introduction}

With the rapid development of society, the health problems of young people are becoming more serious due to the rapid pace of life, high work pressure, and lack of exercise. The sudden death of young people can often be seen in the news, which also reminds young people to exercise more. Indoor fitness equipment such as treadmills, spinning bikes, and fitness chairs have entered millions of households, and smart sports bracelets, smart running shoes, smart weight scales, and sports management apps have gradually become part of people's lives. The introduction of sports auxiliary facilities such as intelligent sports equipment and management software has greatly improved people's interest in sports. At the same time, we also found

*Corresponding author: e-mail: liaofan810@126.com

https://doi.org/10.18494/SAM.2020.2779 
that although existing sports equipment can record the number of steps, the speed of the user, and other data, it is difficult to accurately monitor posture during exercise and give guidance accordingly. However, this also motivated us to design intelligent fitness products using sensors and their related materials and technology.

Sports state recognition provides a technical basis for sports monitoring and providing sports status reminders. ${ }^{(1)}$ Motion state recognition records some motion characteristics of people, infers people's movement state, provides people with exercise suggestions, and even guides people to make health plans. ${ }^{(2)}$ At present, the research on motion state recognition is mainly based on the following two approaches: motion state recognition based on visual equipment and image processing and motion state recognition based on wearable sensors, such as motion sensors, wearable devices with gyroscopes, or other sensors.

In the motion status recognition of pictures and videos, ${ }^{(3,4)}$ a camera is used to take pictures and monitor people in real time, and then image and video analysis technology is used to identify people's activities. However, this type of technology is mainly used in the home monitoring of elderly people and children. Owing to the restrictions of the camera, the camera sensor can only monitor a limited area, which is more useful in identifying problems such as the fall of the person being monitored. ${ }^{(5)}$ However, it is difficult to promote the use of long-term health monitoring and self-quantification of users. ${ }^{(2)}$

In the recognition of wearable sensor motion status, motion status data are collected by attaching wearable sensor devices on people, including acceleration sensors, gyroscopes, and heart rate monitoring devices. Users only need to carry a set of equipment based on widely available sports state recognition technology to collect their own movement data. Mathie et al. ${ }^{(6)}$ described a novel system for objectively and continuously monitoring movement by accelerometry. Biagetti et al. ${ }^{(7)}$ presented a low-cost wearable wireless system specifically designed to acquire surface electromyography (sEMG) and accelerometer signals for monitoring human activity when performing sports and fitness activities, as well as in healthcare applications. $\mathrm{Li}^{(8)}$ combined wearable technology, signal processing technology, and wireless communication technology by using acceleration sensors, biosensors, Bluetooth modules, smartphones, and back-end servers to build a "wearable health monitoring system based on recognition of human movement status" to monitor human movement status. On the basis of real-time recognition, alarms are issued for dangerous fall movements and abnormal physiological signals in different motion states. Han et al. ${ }^{(9)}$ proposed a method of recognizing upper-limb motion gestures for a human-computer interface (HCI) using electronic textile sensors, which consist of a double-layered structure with complementary resistance characteristics. Huang et al. ${ }^{(10)}$ designed a wearable wrist goniometer (WWG) composed of an Arduino Nano and two GY-521 accelerometer-gyroscopes. The WWG can get the carpal postures in six directions and measure actual movement angles.

According to the above references, an acceleration sensor can identify movement state information, ${ }^{(11)}$ and two acceleration sensors can achieve accurate monitoring of wrist movements. ${ }^{(9,10,12)}$ Therefore, to solve the problem of inaccurate monitoring of sports equipment, we have designed an intelligent dumbbell as fitness equipment that incorporates film pressure sensor technology, acceleration sensor technology, a Bluetooth wireless 
communication module, and an Arduino MEGA 2560 control system. Dumbbells have the advantages of occupying a small space, are easy to use, and have fixed movements, making it easy to implement intelligent monitoring.

To accurately monitor dumbbell fitness and increase the interest in fitness exercise, the dumbbells use two acceleration sensors to accurately determine the change in the exercise angle and a film pressure sensor ${ }^{(13)}$ to identify the weight of each dumbbell, determine the intensity of the fitness exercise, and connect to the user's mobile phone via Bluetooth to achieve intelligent management of fitness.

\section{System Structure and Sensors}

To achieve accurate monitoring and the feedback of exercise information, the intelligent dumbbell can automatically determine its weight, actively identify the user's fitness exercise posture, record fitness data, and judge the fitness intensity on the basis of the exercise data to act as a fitness sports monitoring guide. At the same time, users can also set up a fitness mode through their mobile phones to carry out targeted training. The intelligent dumbbell system framework mainly includes seven parts: a film pressure sensor, an acceleration sensor module, an Arduino control board, a voice playback module, an LED display, a wireless transmission module, and a mobile phone app. A block diagram of the system is shown in Fig. 1.

\subsection{Arduino control system}

In order to realize the intelligent dumbbell, the motion data obtained through the acceleration sensor module needs to be processed by the control system. The controller used in the dumbbell is the Arduino MEGA 2560 microcontroller. The microcontroller is an 8-bit microcontroller with 54 digital inputs/outputs, of which 15 can be used as PWM outputs; 16 analog inputs, each with 10-bit resolution; and four UART interfaces to meet the needs of dumbbell status data processing and system control. ${ }^{(14,15)}$

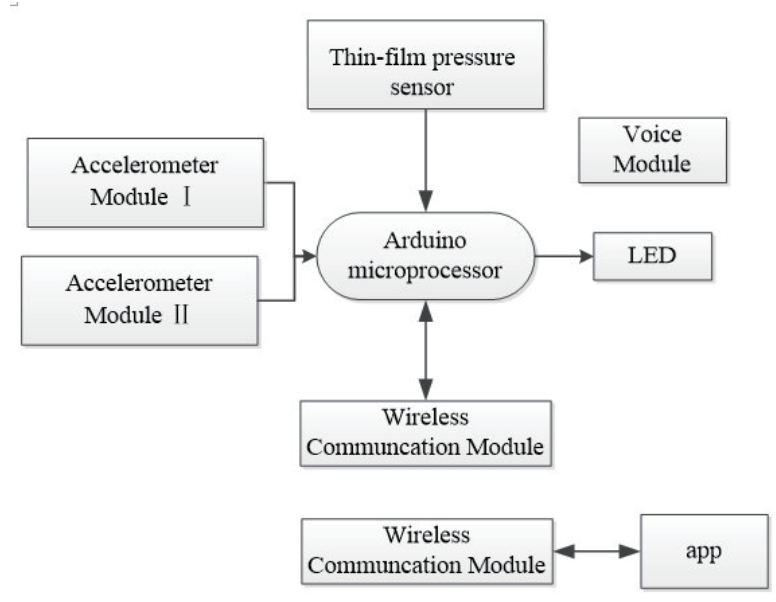

Fig. 1. Block diagram of the intelligent dumbbell. 


\subsection{Thin-film pressure sensor}

As shown in Fig. 2, the thin-film pressure sensor selected for the dumbbell is a piezoresistive flexible pressure sensor. Through the voltage divider circuit in Fig. 2, we can obtain the voltage value of the pressure sensor, and then calculate the weight of the dumbbell.

\subsection{Accelerometer technology}

For the acceleration sensor, we choose the JY16 acceleration sensor module. This module uses a high-precision MPU6050 accelerometer. The processor reads the measurement data of the accelerator and combines the module's internal attitude solver to obtain the threedimensional acceleration, angular velocity, and angle. The single-chip microcomputer can read the measurement data through the serial port connection. The connection diagram is shown in Fig. 3 and the pin functions are shown in Table 1.

To achieve accurate monitoring of dumbbell movements, we designed two acceleration sensors that are installed on both sides of the dumbbell to collect user movement information. By comparing and processing the information from the two accelerator sensors, we can calculate the posture data of the dumbbell, and then judge the user's movement state. Figure 4 shows the acceleration sensor data in the static state.

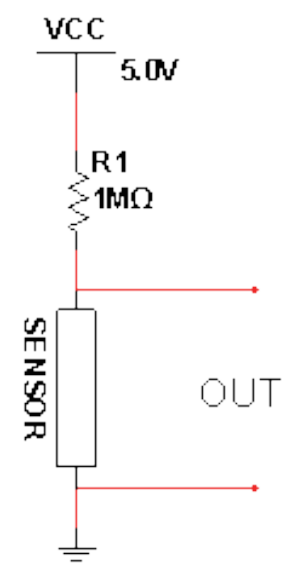

Fig. 2. (Color online) Circuit diagram of thin-film pressure sensor.

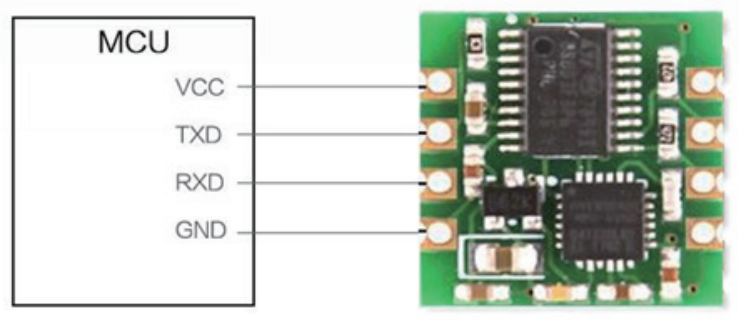

Fig. 3. (Color online) Connection between the acceleration sensor and microcontroller.

Table 1

Interface of the acceleration sensor module.

\begin{tabular}{lc}
\hline Pin & Description \\
\hline VCC & Power supply, 3.3 or $5 \mathrm{~V}$ input \\
RX & Serial data input \\
TX & Serial data output \\
GND & Power supply ground \\
SCL & I2C serial clock \\
SDA & I2C serial data \\
\hline
\end{tabular}




\section{System Design}

\subsection{Hardware}

After deciding the scheme and each part of the module, we designed the hardware circuit. Figure 5 shows the hardware connection of the intelligent dumbbell control system, which includes the above-mentioned module, a music player module, a sound and light alarm module, and buttons.

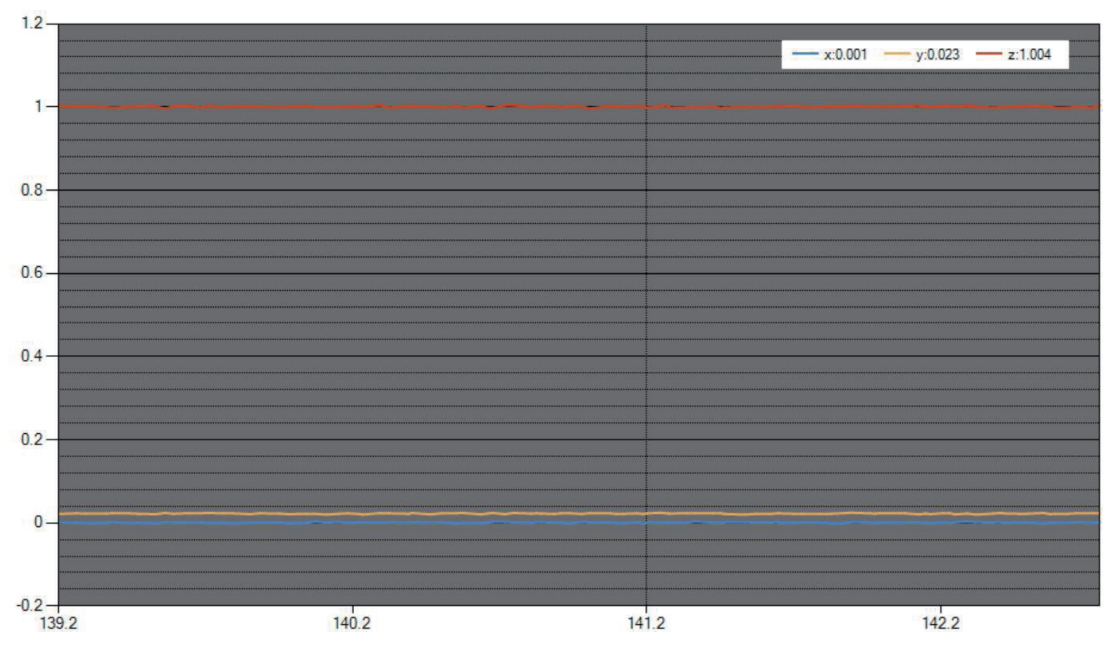

Fig. 4. (Color online) Acceleration sensor data in static state.

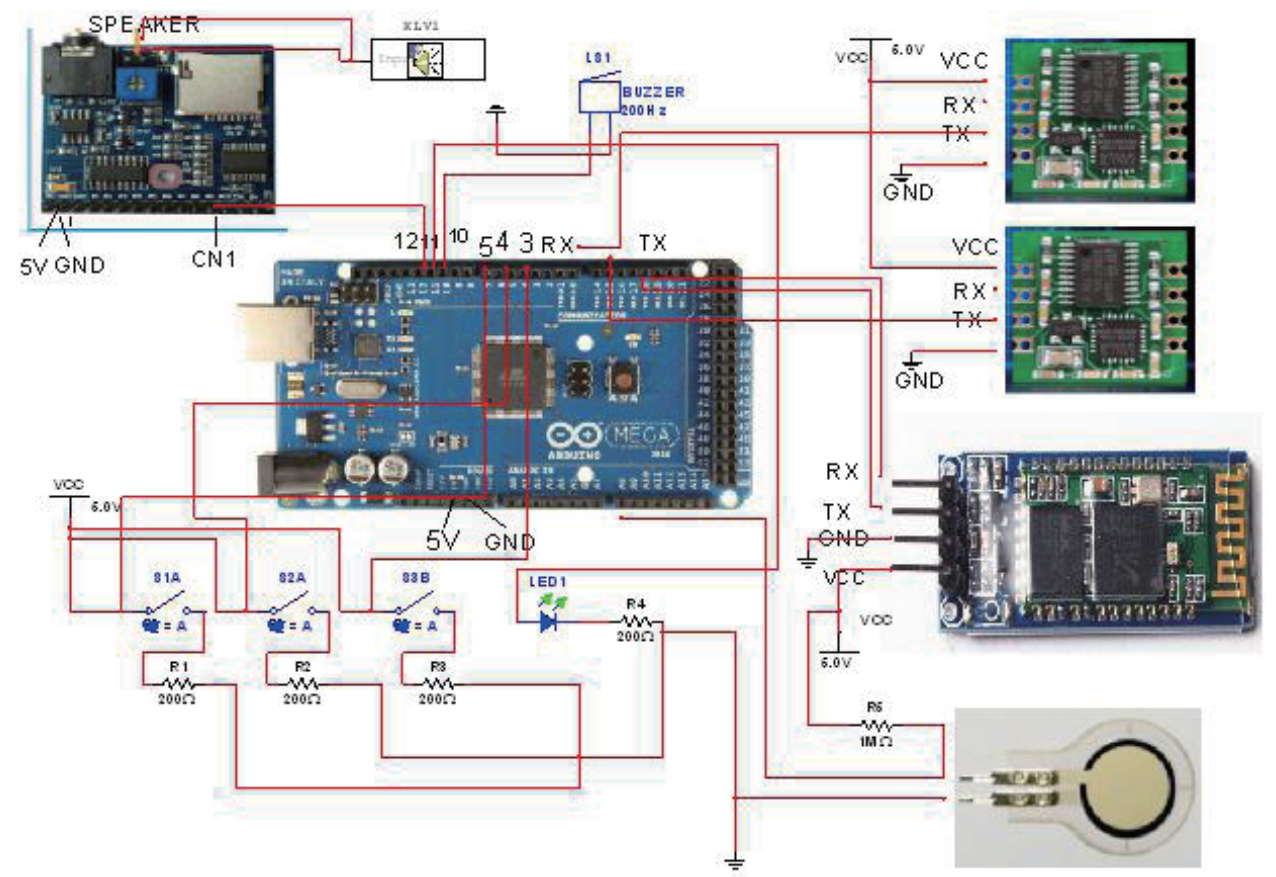

Fig. 5. (Color online) Hardware connection diagram. 


\subsection{Software}

The intelligent dumbbell control system includes two parts: the Arduino information acquisition control system and the mobile phone app. The Arduino information acquisition control system calculates the user's movement status through the dumbbell sensor data, and makes corresponding sound and light prompts, and then transmits this movement status information to the mobile phone. The mobile phone app can realize real-time data monitoring, exercise status judgment and management, and can also control the intelligent dumbbell to play music and give reminders. The flowchart of the microprocessor system is shown in Fig. 6.

\section{Data Analysis and Display}

\subsection{Data analysis}

Dumbbell exercises can be used for strength training and muscle compound training. Muscle training requires fixed standard movements and repeated training, so dumbbell exercises mainly include push, flexion and extension, pull-ups, and curls. To accurately recognize the attitude of the movement, after obtaining the data of the two acceleration sensors, we perform separate calculations to obtain the attitude information, fuse the two sets of data, and compare the characteristic data of different movement states to identify and determine the attitude of the movement. Figures 7-9 show the motion posture information acquired by the sensors under different motion states. As shown in the figures, the $x$-axis is acceleration data during motion, and the $y$-axis is time, and the data of the three axes of each acceleration sensor are displayed in different colors, and different motion states correspond to different acceleration data. Figures 7(a), 8(a), and 9(a) shows the data of the three axes of the acceleration sensor on the left side of

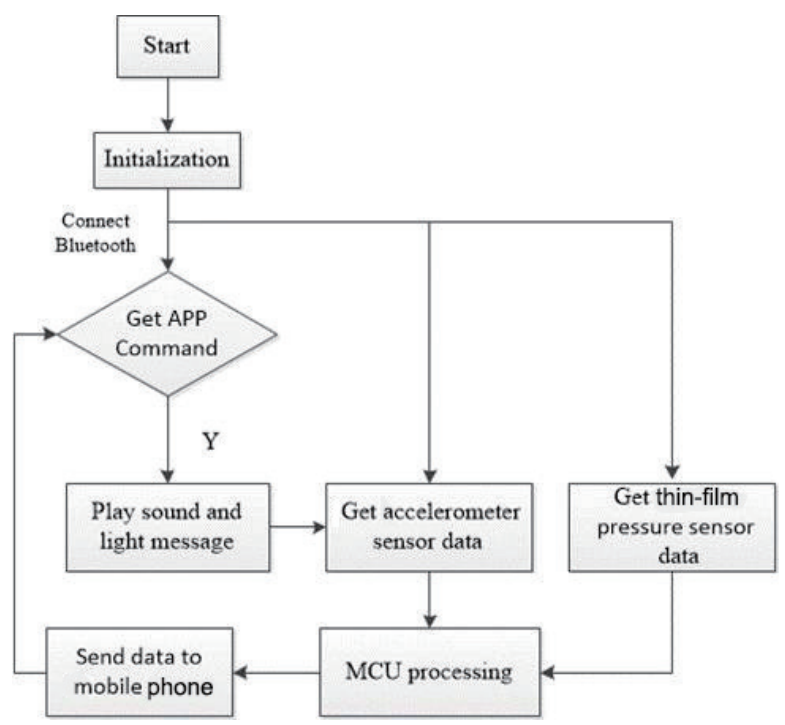

Fig. 6. Flowchart of Arduino microprocessor system. 


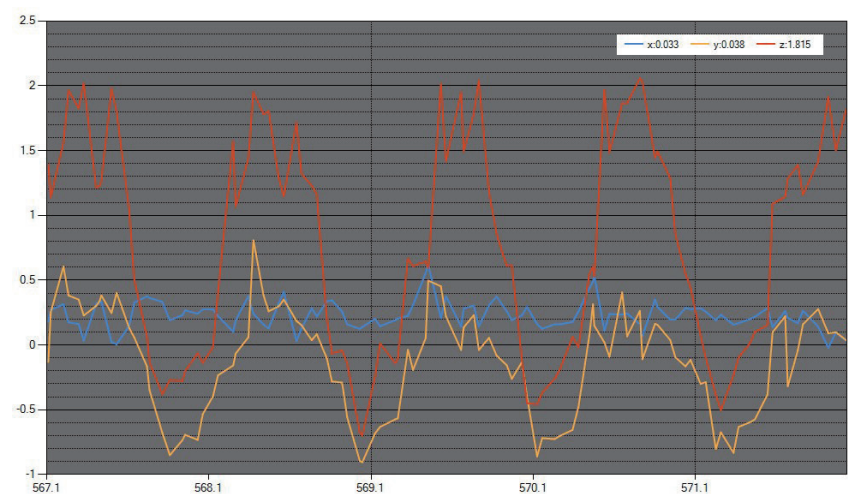

(a)

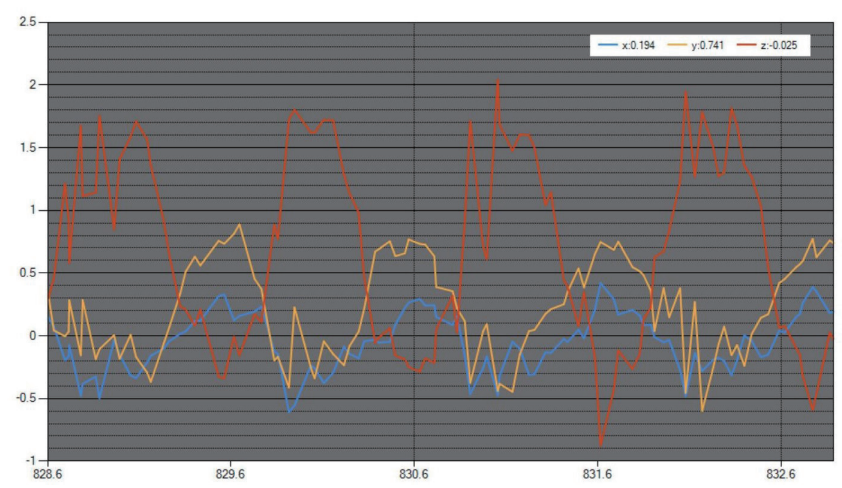

(b)

Fig. 7. (Color online) Horizontal bending motion.

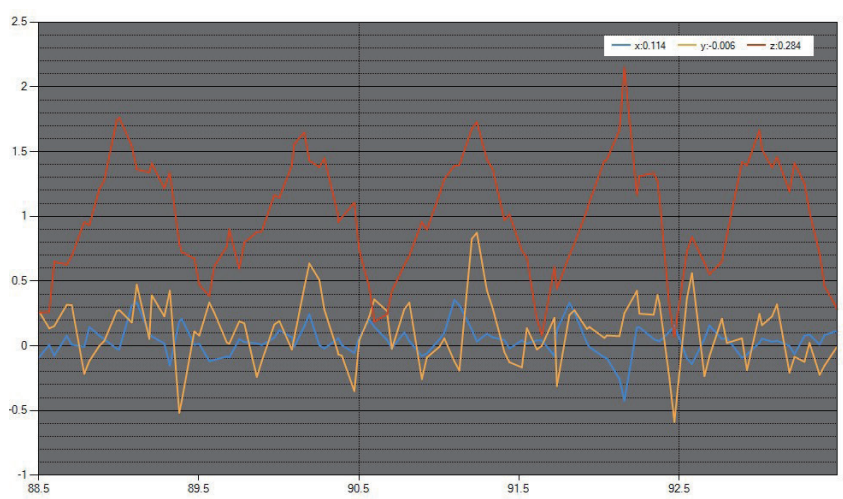

(a)

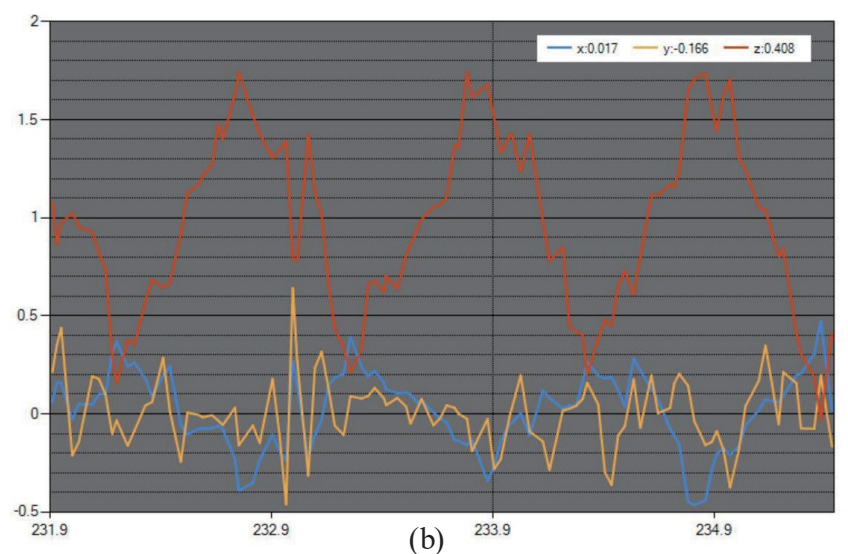

Fig. 8. (Color online) Horizontal push-up motion.

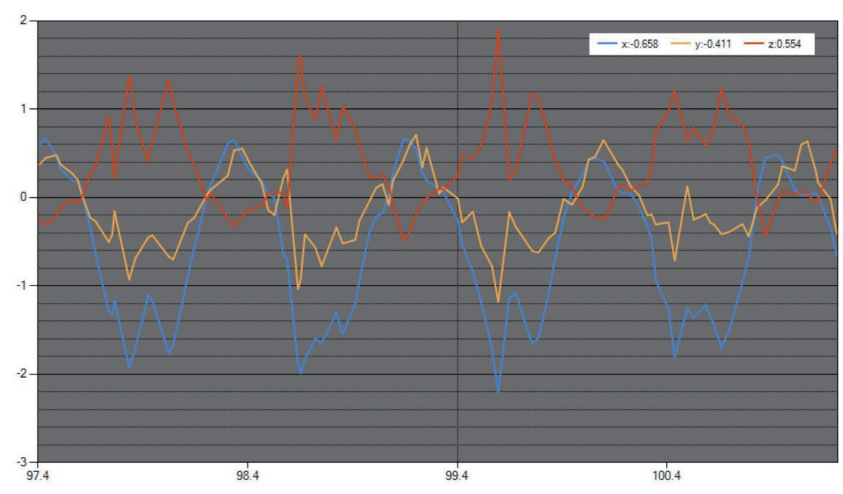

(a)

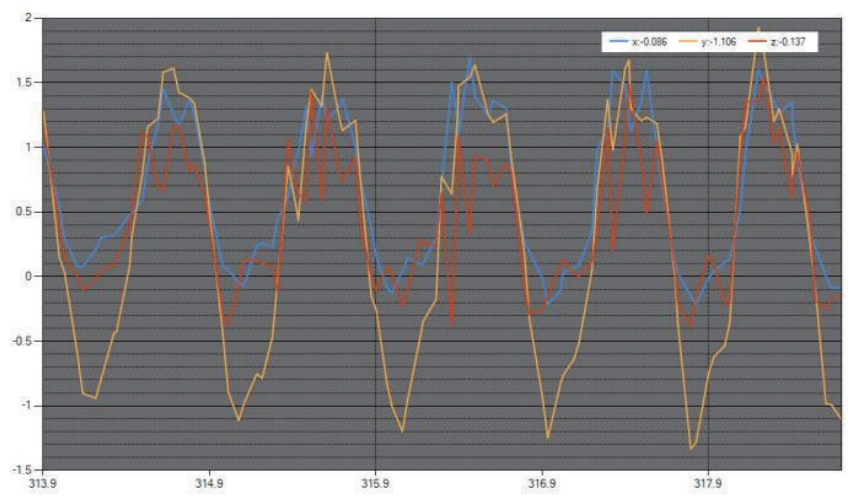

(b)

Fig. 9. (Color online) Vertical bending motion.

the dumbbell, and Figs. 7(b), 8(b), and 9(b) shows the data of the three axes of the acceleration sensor on the right side of the dumbbell. After the sensor installation position is determined, by comparing the two sets of data, we can determine the posture of the dumbbell and then judge the motion state. 


\subsection{Display and interaction}

The app interface (Fig. 10) includes Bluetooth connection, disconnection, start, stop, exercise time, exercise quantity, exercise mode selection, music selection, data recording, and exercise analysis. Through the mobile app, users can obtain dumbbell fitness information and make judgments on fitness activities, such as whether the amount of exercise is too much and whether the posture is standardized, etc., to achieve intelligent guidance of dumbbell fitness.

\subsection{Actual product}

As shown in Fig. 11, the control system is installed on the side of the dumbbell so that the user's movement information can be obtained more accurately.

\section{Results and Discussion}

The intelligent dumbbell can directly measure the pressure data through the pressure sensor and then calculate the dumbbell weight. Through the two symmetrical acceleration sensors, we can obtain the acceleration, angular velocity, and angle information of the acceleration sensors in different states, and the dumbbell status can be recognized by the judgment of the state information. Table 2 shows the attitude data of the dumbbell in the horizontal and vertical states, where $Z$ is the yaw angle, $X$ is the roll angle, and $Y$ is the pitch angle. As shown in Table 2, through the two symmetrical acceleration sensors, we can calculate the angle change in dumbbell movement. We can identify the movement in the horizontal direction through the

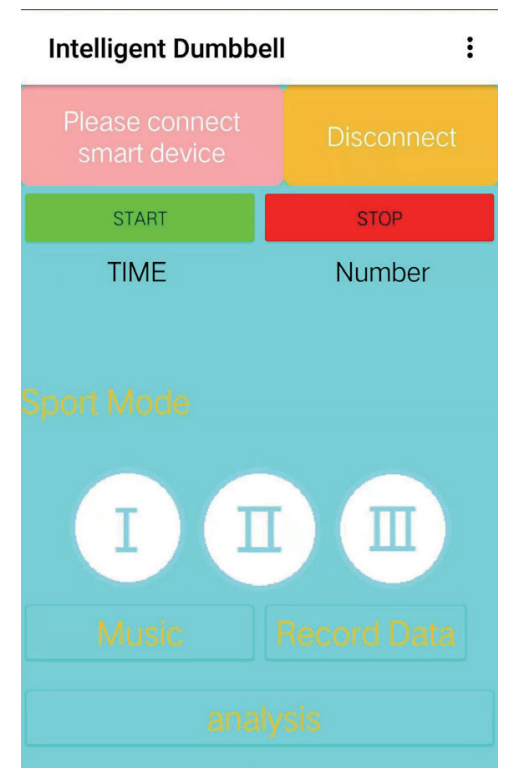

Fig. 10. (Color online) App interface.

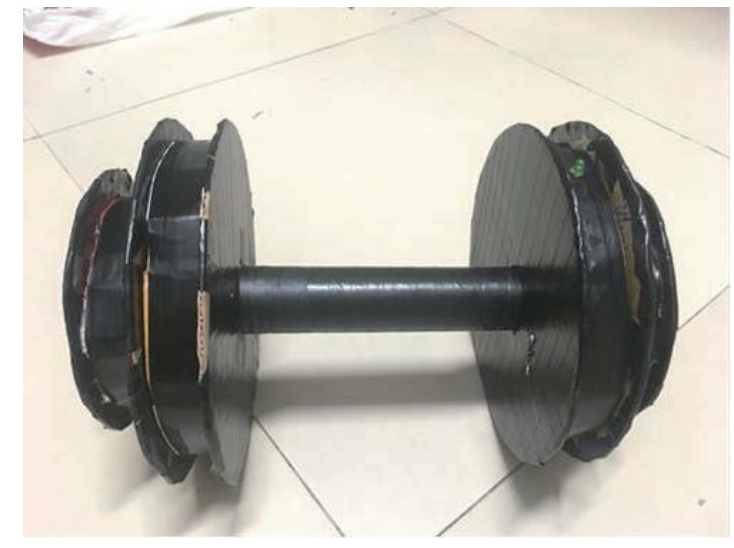

Fig. 11. (Color online) Photograph of intelligent dumbbell. 
Table 2

Attitude data of smart dumbbell.

\begin{tabular}{lccccccc}
\hline & \multicolumn{3}{c}{ Horizontal direction } & & \multicolumn{3}{c}{ Vertical direction } \\
\cline { 2 - 3 } & Angle $X(\mathrm{deg})$ & Angle $Y(\mathrm{deg})$ & Angle $Z(\mathrm{deg})$ & & Angle $X(\mathrm{deg})$ & Angle $Y(\mathrm{deg})$ & Angle $Z(\mathrm{deg})$ \\
\hline $\begin{array}{l}\text { Acceleration } \\
\text { sensor 1 }\end{array}$ & 0.2032 & 4.5209 & -0.6207 & & 59.6173 & -69.4775 & 115.2301 \\
\hline $\begin{array}{l}\text { Acceleration } \\
\text { sensor 2 }\end{array}$ & 4.3066 & 3.8123 & -175.7043 & & 104.5184 & 65.8136 & -119.6686 \\
\hline
\end{tabular}

$Z$ angle data and the movement in the vertical direction through the judgment of the $Y$ angle data. After identifying the horizontal and vertical key data, combined with the acceleration information described in Sect 4.1, the system can accurately give the number and status of dumbbell movements. This paper reports the first ever incorporation of posture recognition and gravity detection technologies into dumbbells, where the characteristics of dumbbell fitness are combined to achieve more detailed fitness monitoring and guidance. This has significance for the development of professional sports equipment design and precise motion-monitoring research.

\section{Conclusions}

Fitness exercise has become popular. However, it has been difficult for traditional fitness equipment to meet people's needs, and intelligent fitness equipment has become a trend. To realize an intelligent dumbbell, we combined a thin-film pressure sensor, an acceleration sensor, an Arduino controller, and Bluetooth wireless communication with a mobile phone app. Its functions include recording the time and frequency of dumbbell exercise, recognizing exercise status, and correcting exercise posture. The dumbbell can basically meet the needs of users' fitness. In the future, we can replace the Arduino controller with other control modules according to requirements to achieve miniaturization and low power consumption, which is expected to be significant for the subsequent development of professional fitness equipment.

\section{References}

1 N. Ravi, N. Dandekar, P. Mysore, and M. Littman: Proc. 12th National Conf. Artificial Intelligence and the Seventeenth Innovative Applications (AAAI, 2005) 1541. https://www.researchgate.net/publication/221604452

2 J. Q. Peng: Master Thesis, Harbin Institute of Technology (2014).

3 P. Turaga, R. Chellappa, V. S. Subrahmanian, and O. Udrea: IEEE Trans. Circuits Syst. Video Technol. 18 (2008) 1473. https://doi.org/10.1109/tcsvt.2008.2005594

4 W. Hu, T. Tan, L. Wang, and S. Maybank: IEEE Trans. Syst. Man Cybern. 34 (2004) 334. https://doi. org/10.1109/TSMCC.2004.829274

5 S. Sukreep, K. Elgazzar, C. H. Chu, C. Nukoolkit, and P. Mongkolnam: Sens. Mater. 31 (2019) 6. https://doi. org/10.18494/SAM.2019.2308

6 M. J. Mathie, J. Basilakis, and B. G. Celler: Proc. 23rd Annu. Int. Conf. IEEE Engineering in Medicine and Biology Society (IEEE, 2001). https://doi.org/10.1109/IEMBS.2001.1019627

7 G. Biagetti, P. Crippa, L. Falaschetti, S. Orcioni, and C. Turchetti: BioMed. Eng. Online 17 (2018) 1. https:// doi.org/10.1186/s12938-018-0567-4

8 N. Li: Master Thesis, Bei Jing University of Technology (2013). 
9 S.-H. Han, E.-J. Ahn, M.-H. Ryu, and J.-N. Kim: Sens. Mater. 31 (2019) 5. https://doi.org/10.18494/ SAM.2019.2261

10 S.-M. Huang, P.-Y. Gi, and P.-Y. Wu: Proc. 2018 IEEE Int. Conf. Advanced Manufacturing (IEEE, 2018). https://doi.org/10.1109/AMCON.2018.8615058

11 L. Sun, D. Q. Zhang, B. Li, B. Guo, and S. J. Li: Proc. 7th Int. Conf. Ubiquitous Intelligence and Computing (UIC, 2010) 548. https://dl.acm.org/doi/10.5555/1929661.1929712

12 S.-M. Huang, P.-Y. Gi, and P.-Y. Wu: Proc. 2018 IEEE Int. Conf. Advanced Manufacturing (IEEE, 2018). https://doi.org/10.1109/AMCON.2018.8615058

13 P. Jonsson and P. W. Johnson: Appl. Ergon. 32 (2001) 599. https://doi.org/10.1016/S0003-6870(01)00036-9

14 J. P. Cui, H. Cao, J. Zhu, J. L. Jiang, and Y. Zhang: China Meas. Test 44 (2018) 8. https://doi.org/10.11857/ j.issn.1674-5124.2018.08.013

15 S.-C. Ho and Y.-C. Wang: Sens. Mater. 31 (2019) 7. https://doi.org/10.18494/SAM.2019.2295

\section{About the Authors}

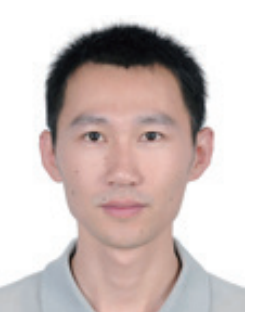

Yang Liu received his B.S. and M.S. degrees in electronic engineering from Northwestern Polytechnical University, Xi'an, China, in 2010 and 2013, respectively. Since 2013, he has worked at Dongguan Polytechnic. His major fields are microprocessor applications, wireless communication, and sensor applications. (liaofan810@126.com)

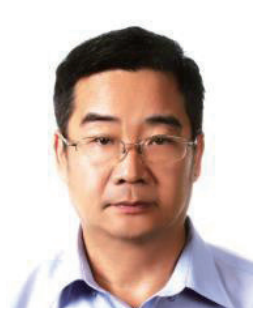

Chun-Liang Hsu received his B.S. degree from National Taiwan Normal University, Taiwan, in 1977 and his M.S. and Ph.D. degrees from the Industry Education Institute, Taiwan, in 1980 and 2000, respectively. From 2000 to 2009, he was an assistant professor at St. John's University, Taiwan. Since 2010, he has been a professor at St. John's University. His research interests are in microsystem technology, embedded systems, wireless communication, and sensors. (liang@mail.sju.edu.tw; xucl@dgpt.edu.cn) 